\title{
Efficiency of adjuvant immunochemotherapy following curative resection in patients with locally advanced gastric cancer
}

\author{
Tadeusz Popiela ${ }^{1}$, Jan Kulig ${ }^{1}$, Antoni Czupryna ${ }^{1}$, Antoni M. Szczepanik ${ }^{1}$, and Marek Zembala ${ }^{2}$
}

${ }^{1}$ First Department of Surgery and Gastrointestinal Surgery, Jagiellonian University Medical College, Cracow, Poland

${ }^{2}$ Department of Clinical Immunology, Jagiellonian University Medical College, Wielicka 265, 30-663 Cracow, Poland

\begin{abstract}
Background. Despite curative resection, $50 \%-90 \%$ of gastric cancer patients die of disease relapse. Although some clinical trials have indicated that chemotherapy and immunochemotherapy may be effective modalities, more recent studies have not been able to define the standard treatment for advanced gastric cancer. The present study evaluated the effect of adjuvant immunochemotherapy with the use of BCG (bacille Calmette-Guérin) and FAM (5-fluorouracil, adriamycin, mitomycin C) chemotherapy on the survival of patients with locally advanced resectable gastric cancer.

Methods. A total of 156 patients with stage III or IV gastric cancer who had undergone curative resection were randomly assigned to three treatment groups: BCG + FAM (immunochemotherapy), FAM (chemotherapy), and control (surgery only). Treatment was continued for 2 years or until death. Further postsurgical follow up was carried on for up to 10 years.

Results. Overall 10-year survival was $47.1 \%$ for the immunochemotherapy group $(P<0.037$ vs FAM and $P<$ $\mathbf{0 . 0 0 0 6}$ vs control), $30 \%$ for the chemotherapy group (vs control, NS), and $\mathbf{1 5 . 2} \%$ for the control group. In patients with pT2/T3 primary tumors, 10-year survival was $55.3 \%$ for BCG + FAM vs $28.2 \%$ for FAM $(P<0.01)$ and $14.6 \%$ for the control group $(P<0.00018)$. BCG + FAM significantly improved the survival of patients with intestinal-type but not diffuse-type cancer. Immunochemotherapy was well tolerated.

Conclusion. This study, based on a limited number of patients, indicates that adjuvant immunochemotherapy (BCG + FAM) may prolong the survival of gastric cancer patients after curative gastrectomy; in particular, in patients with pT2/ T3 tumors and intestinal-type primary tumors. There was no survival benefit from FAM adjuvant chemotherapy.
\end{abstract}

Key word Gastric cancer · Immunochemotherapy · Chemotherapy $\cdot$ BCG $\cdot$ FAM

Offprint requests to: $\mathrm{M}$. Zembala

Received: March 9, 2004 / Accepted: August 18, 2004

\section{Introduction}

Despite improvements in various treatment modalities, gastric cancer has a poor prognosis. Surgical resection of the tumor with the dissection of regional lymph nodes is regarded as the only curative treatment [1]. However, despite curative resection, $50 \%-90 \%$ of patients die of cancer due to disease relapse [1,2]. As residual cancer cells are thought to be responsible for the disease relapse, postsurgical adjuvant chemotherapy has been implemented to prevent recurrence. Various combinations of drugs have been used for adjuvant treatment. The current status of adjuvant chemotherapy is controversial, and only few studies have shown a benefit from this form of treatment. However, the recent Intergroup 0116 study indicates that the combination of 5fluorouracil (5-FU) plus leucovorin chemotherapy, combined with radiotherapy, significantly prolongs overall survival of patients after gastrectomy when compared with no adjuvant treatment [2]. Also, adjuvant chemotherapy with mitomycin C (MMC) alone, or combined with tegafur, improves the survival of patients after curative resection [3]. However, no survival benefit was observed in a phase III trial of adjuvant chemotherapy with MMC, 5-FU, and cytosine arabinoside, followed by oral 5-FU, in serosa-negative gastric cancer patients after curative resection [4].

The present trial was initiated in 1988 to assess the efficacy of postsurgical chemotherapy alone or in combination with immunochemotherapy. At the time of the trial introduction, chemotherapy with 5-FU, adriamycin, and MMC (FAM) was regarded as the most effective for advanced gastric cancer [5] and therefore was used in the adjuvant setting. As intensive chemotherapy may induce long-lasting immunosuppression, which influences the antitumor response of the host [6] the second treatment group received BCG (bacille Calmette-Guérin) together with FAM, in order to assess whether nonspecific immunostimulation may 
Table 1. Clinicopathological characteristics of patients in the treatment groups

\begin{tabular}{|c|c|c|c|}
\hline Characteristics & FAM $(n=53)$ & $\mathrm{BCG}+\mathrm{FAM}(n=51)$ & Control $(n=52)$ \\
\hline $\operatorname{Sex}(M / F)$ & $43 / 10 *$ & $34 / 17$ & $31 / 21$ \\
\hline Age, years $($ mean $\pm \mathrm{SD})$ & $58.4 \pm 11.6$ & $57.2 \pm 11.6$ & $59.6 \pm 10.6$ \\
\hline \multicolumn{4}{|l|}{ Stage III } \\
\hline A & 23 & 21 & 33 \\
\hline B & 15 & 16 & 9 \\
\hline Stage IV (T4N1) & 15 & 14 & 10 \\
\hline \multicolumn{4}{|l|}{ Primary tumor } \\
\hline p T 2-3 & 38 & 37 & 42 \\
\hline p T 4 & 15 & 14 & 10 \\
\hline \multicolumn{4}{|l|}{ Regional lymph nodes } \\
\hline p N1 & 27 & 23 & 35 \\
\hline $\mathrm{p} \mathrm{N} 2$ & 26 & 28 & 17 \\
\hline \multicolumn{4}{|l|}{ Pathological type of cancer } \\
\hline Intestinal & 29 & 27 & $37 * *$ \\
\hline Diffuse & 17 & 16 & 11 \\
\hline Mixed & 7 & 8 & 4 \\
\hline \multicolumn{4}{|l|}{ Location } \\
\hline Upper & 9 & 12 & 11 \\
\hline Middle & 18 & 14 & 20 \\
\hline Lower & 26 & 25 & 21 \\
\hline \multicolumn{4}{|l|}{ Resection } \\
\hline Total & 26 & 29 & 18 \\
\hline Subtotal & 27 & 22 & 34 \\
\hline
\end{tabular}

$* P=0.052 ; * * P=0.041$

affect the overall response. The choice of BCG was indicated by: its effectiveness in the treatment of experimental tumors, some effectiveness of BCG-cell wall skeleton (BCG-CWS) in gastric cancer [7], and the excellent results of intravesical BCG in superficial bladder cancer [8]. Furthermore, our previous trial indicated that the addition of BCG to 5-FU increased the 5-year survival of patients with locally advanced resectable gastric cancer [9].

The aim of the present study was to assess the longterm effect of adjuvant immunochemotherapy (BCG + FAM) vs chemotherapy (FAM) vs control (surgery only) on the survival of patients with locally advanced gastric cancer following curative resection.

\section{Patients and methods}

In the 5 years prior to the trial design, the average number of gastric cancer patients treated at the Department per year was approximately 100 , and one-third of them were estimated as being candidates for entering the trial. Therefore, we decided to recruit patients for 5 years to achieve the number of 50 patients in each arm. With such a sample size, and expected differences in survival between groups of $25 \%$, as well as a follow-up time of 5 years, this study achieved a power of $80 \%$ at a 0.05 significance level, calculated for the two-sided log rank test.
Patients with biopsy-proven carcinoma of the stomach who had been operated on between 1988 and 1992, and who were classified, after postsurgical clinicopathological workup, according to the International Union Against Cancer (UICC) TNM system [10], as having stage III or IVA disease entered the prospective, randomized (by an envelope method) trial. To make the analysis comparable to the results of other trials, reclassification of patients was performed, using the currently approved staging system (UICC: TNM Classification of malignant tumors $5^{\text {th }}$, ed., 1997[11]). Patients with stage IIIA, IIIB, or IV (T4N1M0) gastric cancer were included (Table 1). No patient was excluded during the reclassification.

All patients underwent R0/R1 curative gastrectomy with D2 lymph node, i.e., N1 and N2, dissection [12]. Indications for total stomach resection were: diffuseand mixed-type cancer according to Lauren's classification [13], and intestinal-type cancer of the middle and upper stomach with a proximal margin of less than $5 \mathrm{~cm}$ [12]. The inclusion criteria for eligibility were: patients below the age of 70 years, WHO performance status of less than 2, and expected survival of 12 months or more, with no previous treatment. Patients with low leukocyte $\left(<3 \times 10^{9} / 1\right)$ or platelet $\left(<1 \times 10^{11 / 1}\right)$ levels, or elevated alanine and aspartate transaminases were excluded. The eligible patients were randomly assigned postoperatively to three treatment groups: BCG + FAM, FAM, and no further treatment (control group). 
The treatment protocol was approved by the Ethics Committee of the Jagiellonian University Medical College, and informed consent was obtained from each patient or the patient's family. Adjuvant therapy started within 21-35 days after surgery. Of the 164 eligible patients, 8 refused further treatment and were excluded from randomization. None of the 156 randomized patients withdrew their consent.

Chemotherapy consisted of the FAM regimen: 5-FU, adriamycin, and MMC. All drugs were given intravenously. 5-FU, at the dose of $600 \mathrm{mg} / \mathrm{m}^{2}$, was delivered on days $1,8,29$, and 36 ; adriamycin, $30 \mathrm{mg} / \mathrm{m}^{2}$, on days 1 and 29; and MMC, $10 \mathrm{mg} / \mathrm{m}^{2}$, on day 1 of the cycle [5]. Each course of treatment was given every 8 weeks. Adriamycin was stopped at a total cumulative dose of $600 \mathrm{mg}$. Fresh frozen BCG, Moreau strain (Sera and Vaccine Laboratory, Lublin, Poland) was administered by skin scarification to deliver $2-4 \times 10^{8}$ viable units per dose, as previously described [9]. BCG treatment started within 2 weeks from the beginning of chemotherapy and was given every week during the first 3 months and biweekly thereafter. Treatment was continued for 2 years or until death, unless withdrawn because of unacceptable side effects.

Patients were treated at the outpatient clinic of a single institution, and their performance status recorded during each course of therapy. A full clinical assessment (physical, endoscopic, radiological, and ultrasonographic, as applicable) was performed every 6 months during the treatment period and every 12 months thereafter. A complete blood count, and blood and urine biochemistry were recorded at the beginning of each cycle of chemotherapy. When the leukocyte count was below $2 \times 10^{9} / 1$, platelets were below $5 \times 10^{11} /$ 1 , or transaminases were more than twofold elevated, the therapy was suspended (usually for less than 6 weeks) until the toxic effects subsided. Side effects were classified according to Southwest Oncology Group (SWOG) criteria [14].

The primary endpoint of this study was the duration of survival [15]. Overall survival was defined as the time between the date of surgery and the date of death. Survival curves were calculated by the method of Kaplan-Meier, while statistical comparisons were made by the two-sided log rank test [15]. The median survival was also analyzed. Differences between the groups were estimated using the $\chi^{2}$ test (Statistica program 5.0 PL; StatSoft, Tulsa, OK, USA), and a $P$ value of less than 0.05 was regarded as statistically significant. The hazard ratio and its $95 \%$ confidence interval (CI) were employed for comparison of the treatment groups. Retrospective stratification according to the Cox proportional hazard regression model was used to adjust for possible underlying variables.

\section{Results}

There were no major differences between the treatment groups with regard to clinicopathological characteristics (Table 1). However, there were some imbalances in the control group: there was a higher proportion of patients with N1 involvement compared with the other two groups (NS), and a higher proportion of patients with the intestinal type of cancer according to the pathological classification of Lauren $(P=0.041)$, who, in consequence, underwent subtotal resection. A higher proportion of male patients was present in the chemotherapy (FAM) group $(P=0.052)$ compared with the other two groups.

Treatment compliance was good, although the immunochemotherapy group had more chemotherapy cycles administered (mean, 10.1) than the chemotherapy group (mean, 8.5). This was probably due to differences between these groups in the mortality rate during the first 2 years, i.e., when treatment was applied (proportion of deaths, in BCG + FAM/FAM, 74\%; proportion of cycles, $84 \%$ ).

The overall survival at 10 years was $47.1 \%$ for the immunochemotherapy group, $30 \%$ for the chemotherapy group, and $15.2 \%$ for the control group (Fig. 1A). The differences between the BCG + FAM group and the two other groups were statistically significant $(P$ $<0.0006$ for difference from controls and $P<0.037$ for difference from FAM), while no difference between the chemotherapy and the control group was observed. The median survival time for the BCG + FAM group was 85.3 months, whereas it was 27.7 months for the FAM group and 26.6 months for the control group. The hazard ratio for BCG + FAM vs FAM was 0.76 (95\% CI, $0.54-1.03 ; P=0.08)$, that for BCG + FAM vs control was $0.62(95 \% \mathrm{CI}, 0.45-0.81 ; P=0.001)$, and that for FAM vs control was 0.82 (95\% CI, $0.65-1.01 ; P=$ $0.07)$. Multivariate analysis of the independent prognostic factors (Cox proportional hazard model) indicated that age and $\mathrm{N}$ status had a significant impact on survival, although they were not different between the treatment groups.

The differences in 10-year survival were even more significant than differences in overall survival: $55.3 \%$ for the BCG + FAM group, $28.2 \%$ for the FAM group, and $14.6 \%$ for the control group when patients with pT2/T3 primary tumors were analyzed (Fig. 1B). No difference was observed in patients with T4 tumor (not shown).

The effect of treatment on the survival of the subgroups of patients with intestinal type and diffuse types of tumors was also analyzed (Fig. 2A, B). BCG + FAM significantly improved the survival (51.8\% at 10 years) of patients with intestinal-type cancer in comparison to the control group $(P<0.004)$ and the FAM group $(P<$ 

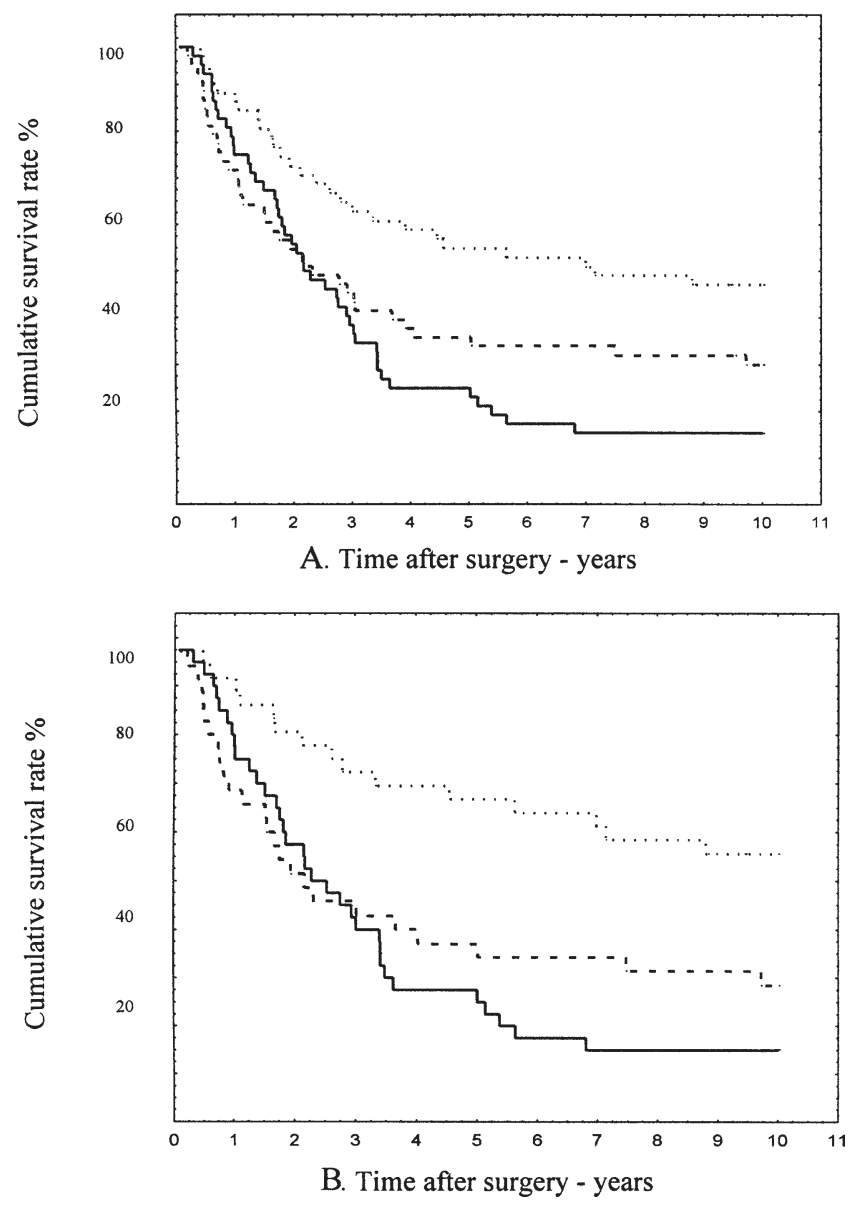

Fig. 1A,B. Overall survival (A) and survival of patients with T2/T3 primary tumor (B). Continuous lines, control group; dashed lines, FAM group (5-fluorouacil, adriamycin, and mitomycin C); dotted lines, BCG (bacille Calmette-Guérm) + FAM group. A Control vs FAM, NS; control vs BCG + FAM, $P<0.0006$; FAM vs BCG + FAM $P<0.037$. B Control vs FAM, NS; control vs BCG + FAM, $P<0.00018$; FAM vs BCG + FAM, $P<0.01$

0.039). However, BCG + FAM had no significant effect in patients with diffuse-type cancer (Fig. 2B).

Adverse side effects according to the SWOG II/III criteria were infrequent, and consisted of myelosuppression, and cardio-, nephro-, or hepatotoxicity (Table 2). Striking differences were observed in the number of leukopenia episodes, which were significantly less common in the immunochemotherapy group. The other side effects were distributed similarly between the treatment groups, except for elevated transaminases, which were more frequent in the chemotherapy group. Among patients receiving BCG, 19 episodes of strong, short-lasting local inflammatory reactions, usually at the beginning of therapy, and raised temperature (up to $38^{\circ} \mathrm{C}$ ) were noted. These reactions usually required no medication. However, for some patients, paracetamol or metamisol was given.

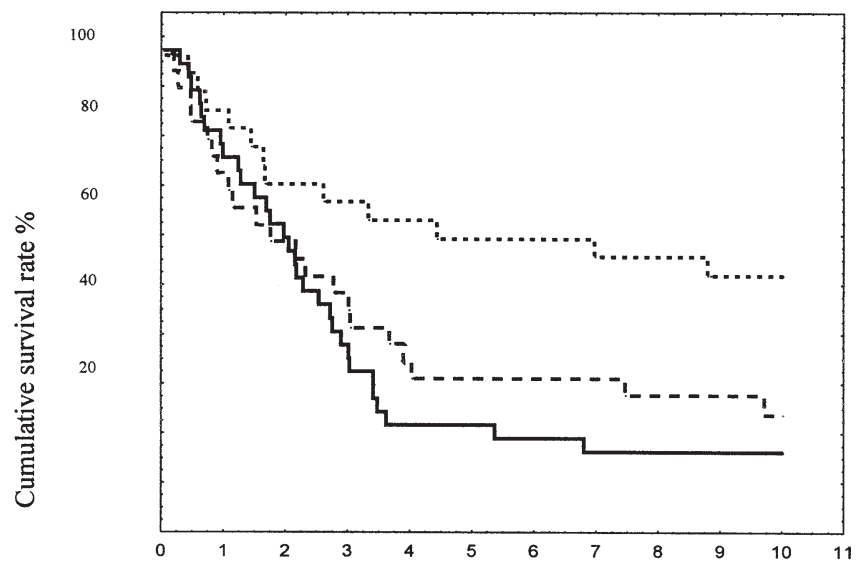

A. Time after surgery - years

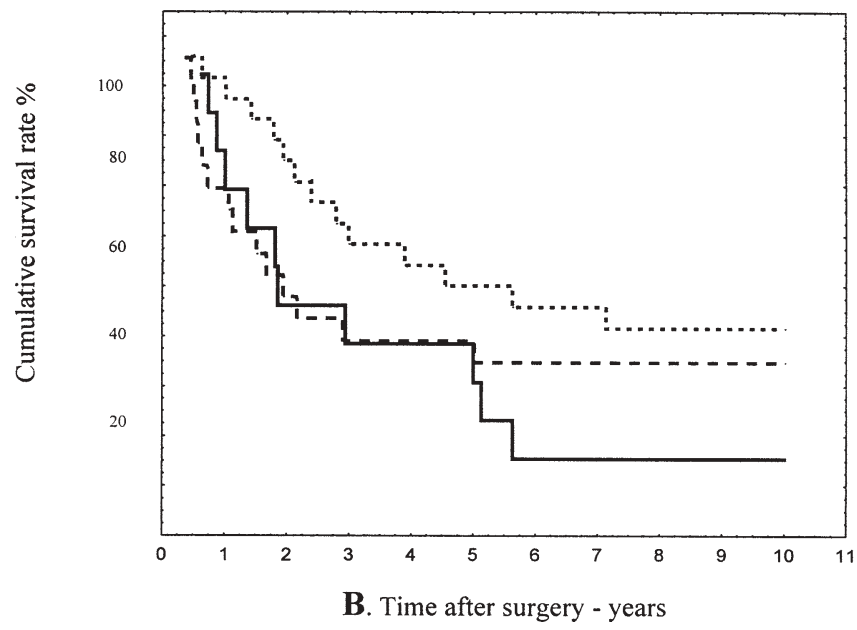

Fig. 2A,B. Survival of patients with intestinal (A) and diffuse/ mixed (B) types of tumors. Continuous, dashed, and dotted lines, as in Fig. 1. A Control vs FAM, NS; control vs BCG + FAM, $P<0.00435$; FAM vs BCG + FAM, $P<0.03942$. B Control vs FAM, NS; control vs BCG + FAM, NS; FAM vs $\mathrm{BCG}+\mathrm{FAM}, \mathrm{NS}$

\section{Discussion}

This study demonstrates that the addition of BCG as an immunostimulant to adjuvant FAM chemotherapy significantly prolongs the survival of patients with advanced gastric cancer who have undergone curative resection. The reduction of about $17 \%$ in the death rate in comparison to that in the group with FAM therapy and about $32 \%$ in comparison to the control group is probably clinically significant. The effect on the mortality rate of the addition of BCG as an immunostimulant is almost identical to the $20 \%$ decreased mortality rate observed in a large Japanese trial in which PSK (protein-bound polysaccharide from Coriolus versicolor) was added as an immunostimulant to the standard chemotherapy (MMC + oral 5-FU) for adjuvant treatment of patients with gastric cancer after curative 
Table 2. Adverse events of adjuvant therapy: number of episodes during the total treatment period

\begin{tabular}{lcc}
\hline SWOG II/III criteria & FAM $(n=53)$ & BCG + FAM $(n=51)$ \\
\hline Leucopenia & $126^{*}$ & 45 \\
Thrombocytopenia & 4 & 5 \\
Cardiotoxicity & 1 & 3 \\
Nephrotoxicity & 20 & 24 \\
Hepatotoxicity & 17 & 10 \\
Local complications & 1 & 19 \\
$\quad$ (site of administration) & & \\
$* P<0.001$ & &
\end{tabular}

resection [16]. In our study, comparison of the survival of patients treated with FAM and that of the control group receiving no further treatment revealed no effect of adjuvant chemotherapy. This is in keeping with other data suggesting the lack of effect of postsurgical adjuvant chemotherapy $[1,17,18]$. In particular, MacDonald et al. [18] found that FAM was not effective adjuvant therapy for stage I-III resectable gastric cancer. It is of interest that, in the present trial, the 5-year survival of our FAM and control groups ( $25 \%$ and $34 \%$ ) was very similar to the overall survival of $32 \%$ found in a large European Organization for Research and Treatment of Cancer (EORTC) trial (no. 40813) [17]. That result, taken together, with ours, suggests that FAM chemotherapy cannot be advocated as a standard adjuvant treatment for locally advanced gastric cancer.

Our analysis of the subsets of patients with T2/T3 (Fig. 1B) vs T4 (not shown) primary tumors and intestinal versus diffuse pathological types of cancer revealed that T2/T3 and intestinal-type tumors, but not T4 or diffuse-type tumors, responded to immunochemotherapy. Although this analysis must be treated with caution, as these subsets of patients may have not been compatible with regard to other variables, these findings seem to suggest that immunochemotherapy may be effective only in patients with small primary tumor with intestinal-type histology. The response of patients with T2/T3 tumors was observed in another immunochemotherapy trial (PSK + MMC + oral 5-FU vs chemotherapy alone) in 262 patients after curative resection [16]. In that trial, the 5-year survival rates were $70.0 \%$ for the immunochemotherapy group and $60 \%$ for the chemotherapy group. Furthermore, we also found that BCG + FAM treatment had no impact on the survival of patients with resectable stage T3N3 and T4N2 gastric cancer (not shown).

Immunochemotherapy was generally well tolerated, and no major differences in the side effects were noted in comparison to the chemotherapy group. However, some patients experienced a strong inflammatory reaction at the site of BCG administration, often accompa- nied by increased body temperature. These events were usually observed at the beginning of treatment, and subsided during subsequent applications. A notable difference between the treatment groups was a significantly lower incidence of leukopenia in patients receiving the BCG + FAM therapy. The reason for this is unclear, but it could be due to the effect of granulocyte macrophage colony-stimulating factor (GM-CSF), which is known to be induced by BCG [19]. It is debatable whether this was an important factor that allowed a better tolerance of chemotherapy, resulting in the increased number of cycles of FAM therapy that these patients received. This increased number of cycles of FAM may be supported by findings that patients receiving GM-CSF tolerated a higher dose of chemotherapy [20].

The nonspecific immunotherapy initiated by Mathé [21] with the use of BCG has been largely abandoned due to a lack of consistent effects. However, several studies have demonstrated its effectiveness in gastric cancer. Thus, BCG-CWS and 5FU, MMC, arabinside cytosine (MFC) [7] or PSK and carbazilquinone [22] immunochemotherapy significantly prolonged the survival of patients with operable gastric cancer. Similarly, postoperative immunochemotherapy with the use of the streptococcal lysate OK-432 was effective in patients with gastric cancer and serosal invasion [23]. Our previous observations suggested that the addition of BCG to 5-FU improved the survival of patients with stage III gastric cancer, even though the results were inferior in comparison to those of the present trial [9]. However, immunochemotherapy was not effective in other types of cancer [24], which raises the question as to whether gastric cancer and superficial bladder cancer [25] are exceptions. The reasons for the beneficial effects of BCG immunotherapy are unclear, and it is possible that several factors may influence the outcome of this treatment [19]. These may include: the production of tumor necrosis factor alpha (TNF) and other antitumor cytokines, as observed in bladder cancer treated with BCG [26], the enhancement of 
monocyte- and lymphocyte - mediated tumor cellkilling [27] synergism, or the better toleration of chemotherapy. TNF may play a special role in the local antitumor response, as our observations suggest that, when given intra-tumorally before surgery, TNF may prolong the survival of patients with locally advanced resectable gastric cancer [28], while the systemic treatment of patients with gastric cancer with BCG increases the antitumor response of monocytes, as measured by TNF production [19].

We conclude that adjuvant immunochemotherapy consisting of BCG + FAM may be effective in the prolongation of survival in patients with locally advanced gastric cancer who have undergone curative gastrectomy; in particular, in patients with T2/T3 and intestinal-type tumors. However, these results must be treated with caution due to the limited number of patients in the trial.

Acknowledgments This study was supported by the State Committee for Scientific Research (grants no. 0671/PO5/2001/20, PBZ-KBN-083/PO5/2002 and PBZ$\mathrm{KBN}-091 / \mathrm{P} 5 / 2003)$.

\section{References}

1. Alberts SR, Cervantes A, van de Velde CJ. Gastric cancer: epidemiology, pathology and treatment (Review). Ann Oncol 2003; 14(Suppl 2):31-6.

2. Macdonald JS, Smalley SR, Benedetti J, Hundahl SA, Estes NC, Stemmermann GN, et al. Chemoradiotherapy after surgery compared with surgery alone for adenocarcinoma of the stomach or gastroesophageal junction. N Engl J Med 2001;345:725-30.

3. Grau JJ, Martin M, Fuster J, Pera M, Garcia-Valdecasas JC, Bombi JA, et al. Impact of adjuvant chemotherapy in the longterm outcome of patients with resected gastric cancer. J Surg Oncol 2003;82:234-40.

4. Nashimoto A, Nakajima T, Furukawa H, Kitamura M, Kinoshita T, Yamamura Y, et al. Randomized trial of adjuvant chemotherapy with mitomycin, fluorouracil, and cytosine arabinoside followed by oral fluorouracil in serosa-negative gastric cancer: Japan Clinical Oncology Group 9206-1. J Clin Oncol 2003;21:2282-7.

5. Macdonald JS, Schein PS, Wooley PV. 5 Fluorouracil, doxorubicin, mitomycin C (FAM) combination chemotherapy for advanced gastric cancer. Ann Intern Med 1980;93:533-6.

6. Mackall CL, Fleisher TA, Brown MR, Andrich MP, Chen CC, Feuerstein IM, et al. Distinctions between $\mathrm{CD}^{+}$and $\mathrm{CD} 4^{+} \mathrm{T}$-cell regenerative pathways result in prolonged T-cell subset imbalance after intensive chemotherapy. Blood 1997;89:3700-7.

7. Ochiai T, Sato H, Hayashi R, Asano T, Sato H, Yamamura Y. Postoperative adjuvant immunotherapy of gastric cancer with BCG cell wall skeleton. Cancer Immunol Immunother 1983;14: 167-71.

8. Lamm DL. Optimal BCG treatment of superficial bladder cancer as defined by American trials. Eur Urol 1992;21:12-6.

9. Popiela T, Zembala M, Oszacki J, Jȩdrychowski W. A follow-up study on chemoimmunotherapy (5-fluorouracil and BCG) in advanced gastric cancer. Cancer Immunol Immunother 1982;13: $182-4$.

10. Fielding JW, Roginski C, Ellis DJ, Jones BG, Powell J, Waterhouse JA, et al. Clinicopathological staging of gastric cancer. Br J Surg 1984;71:677-80.
11. Hermanek P, Sobin LH. UICC: TNM Classification of malignant tumours. 5th ed. Berlin Heidelberg New York Tokyo: Springer; 1997. pp. 24-5.

12. Maruyama K, Okabayashi K, Kinoshita T. Progress in gastric cancer surgery in Japan and its limits of radicality. World J Surg 1987;11:418-25.

13. Lauren P. The two histological main types of gastric carcinoma: diffuse and so-called intestinal-type carcinoma. An attempt at histoclinical classification. Acta Pathol Microbiol Scand 1965;64: $31-42$.

14. Green S, Heiss GR. Southwest Oncology Group Standard Response Criteria, end point definition, and toxicity criteria. Invest New Drugs 1992;10:239-53.

15. Peto R, Pike MC, Armitage P, Breslow NE, Cox DR, Howard SV, et al. Design and analysis of randomised clinical trial requiring prolonged observation of each patient. II Analysis and examples. Br J Cancer 1977;35:1-39.

16. Nakazato H, Koike A, Saji S, Ogawa N, Sakamoto J. Efficacy of immunochemotherapy as adjuvant treatment after curative resection of gastric cancer. Lancet 1994;343:1122-6.

17. Lise M, Nitti D, Marchet A, Sahmoud T, Buyse M, Duez N, et al. Final results of a phase III clinical trial of adjuvant chemotherapy with the modified fluorouracil, doxorubicin, and mitomycin regimen in resectable gastric cancer. J Clin Oncol 1995;13:275763.

18. Macdonald JS, Fleming TR, Peterson RF, Berenberf JL, McClure $\mathrm{S}$, Chapman RA, et al. Adjuvant chemotherapy with 5-FU, adriamycin, and mitomycin-C (FAM) versus surgery alone for patients with locally advanced gastric adenocarcinoma: Southwest Oncology Group study. Ann Surg Oncol 1995;2:488-94.

19. Zembala M, Czupryna A, Więckiewicz J, Jasiński M, Pryjma J, Ruggiero I. Tumour-cell induced production of tumour necrosis factor by monocytes of gastric cancer patients receiving BCG immunotherapy. Cancer Immunol Immunother 1993;36:127-32.

20. Bajetta E, Di Bartolomeo M, Carnaghi C, Buzzoni R, Mariani L, Gebbia V, et al. FEP regimen (epidoxorubicin, etoposide and cisplatin) in advanced gastric cancer, with or without low-dose GM-CSF: an Italian Trial in Medical Oncology (ITMO) study. Br J Cancer 1998;77:1149-54.

21. Mathé G. Systemic active immunotherapy is shifting from middle ages to renaissance period. I. The multiplication of randomized trials showing significant effect of active immunotherapy on residual minimal disease. Cancer Immunol Immunother 1978;35: 149-52.

22. Kondo T, Sakamoto J, Nakazato H. Alternating immunochemotherapy of advanced gastric carcinoma: a randomized comparison of carbazilquinone and PSK to carbazilquinone in patients with curative gastric resection. Biotherapy 1991;3:28795.

23. Maehara Y, Okuyama T, Kakeji Y, Baba H, Furusawa M, Sugimachi K. Postoperative immunochemotherapy including streptococcal lysate OK-432 is effective for patients with gastric cancer and serosal invasion. Am J Surg 1994;169:36-40.

24. Buzdar AU, Blumenschein GR, Smith TR. Adjuvant chemotherapy with fluorouracil, doxorubicin and cyclophosphamide, with or without BCG and with or without irradiation in operable breast cancer. Cancer 1984;53:384-9.

25. Jackson AM, James K. Understanding the most successful immunotherapy for cancer. Immunologist 1994;2:208-15.

26. Pryor K, Goddard J, Golstein D, Stricker P, Russel P, Golovsky D. BCG enhances monocyte- and lymphocyte-mediated bladder tumour cell killing. Br J Cancer 1995;71:801-07.

27. Fujimoto T, Duda RB, Szilvasi A, Chen X, Mai M, O'Donnell MA. Streptococcal preparation OK-432 is a potent inducer of IL12 and a T helper cell 1 dominant state. J Immunol 1997;158:561926.

28. Karcz D, Popiela T, Szczepanik AM, Czupryna A, Szymańska B, Siedlar M, et al. Preoperative endoscopic intratumour application of TNF alpha in patients with locally advanced resectable gastric cancer. Endoscopy 1994;26:369-70. 